\title{
BOUNDED APPROXIMATION PROPERTIES IN TERMS OF $C[0,1]$
}

\author{
ÅSVALD LIMA, VEGARD LIMA and EVE OJA*
}

\begin{abstract}
Let $X$ be a Banach space and let $\mathscr{I}$ be the Banach operator ideal of integral operators. We prove that $X$ has the $\lambda$-bounded approximation property $(\lambda$-BAP) if and only if for every operator $T \in \mathscr{I}\left(X, C[0,1]^{*}\right)$ there exists a net $\left(S_{\alpha}\right)$ of finite-rank operators on $X$ such that $S_{\alpha} \rightarrow I_{X}$ pointwise and

$$
\limsup _{\alpha}\left\|T S_{\alpha}\right\|_{\mathscr{I}} \leq \lambda\|T\|_{\mathscr{I}} .
$$

We also prove that replacing $\mathscr{I}$ by the ideal $\mathscr{N}$ of nuclear operators yields a condition which is equivalent to the weak $\lambda$-BAP.
\end{abstract}

\section{Introduction}

Let $X$ and $Y$ be Banach spaces. We denote by $\mathscr{L}(X, Y)$ the Banach space of all bounded linear operators from $X$ to $Y$, and we write $\mathscr{L}(X)$ for $\mathscr{L}(X, X)$. The subspace of $\mathscr{L}(X)$ of finite-rank operators is denoted by $\mathscr{F}(X)$. Let $I_{X}$ denote the identity operator on $X$.

Recall that a Banach space $X$ is said to have the approximation property (AP) if there exists a net $\left(S_{\alpha}\right) \subset \mathscr{F}(X)$ such that $S_{\alpha} \rightarrow I_{X}$ uniformly on compact subsets of $X$. If $\left(S_{\alpha}\right)$ can be chosen with $\sup _{\alpha}\left\|S_{\alpha}\right\| \leq \lambda$ for some $\lambda \geq 1$, then $X$ is said to have the $\lambda$-bounded approximation property ( $\lambda$-BAP).

Let $\mathscr{A}=\left(\mathscr{A},\|\|_{\mathscr{A}}\right)$ be a Banach operator ideal. Recently, an approximation property which is bounded for $\mathscr{A}$ was introduced and studied in [11] as follows. We say that $X$ has the $\lambda$-bounded approximation property for $\mathscr{A}$ ( $\lambda$-BAP for $\mathscr{A})$ if for every Banach space $Y$ and every operator $T \in \mathscr{A}(X, Y)$ there exists a net $\left(S_{\alpha}\right) \subset \mathscr{F}(X)$ such that $S_{\alpha} \rightarrow I_{X}$ uniformly on compact subsets of $X$ and

$$
\limsup _{\alpha}\left\|T S_{\alpha}\right\|_{\mathscr{A}} \leq \lambda\|T\|_{\mathscr{A}} \text {. }
$$

The $\lambda$-BAP for $\mathscr{A}$ extends the notion of the weak $\lambda$-BAP which is, by definition, the $\lambda$-BAP for the ideal $\mathscr{W}$ of weakly compact operators. The weak

\footnotetext{
* The research of Eve Oja was partially supported by Estonian Science Foundation Grant 7308 and Estonian Targeted Financing Project SF0180039s08.

Received 14 July 2010, in final form 29 October 2010.
} 
BAP was introduced in [12] and studied in [11], [12], [13], [17], [18], [19], [20]. It is immediate that the $\lambda$-BAP implies the $\lambda$-BAP for every Banach operator ideal $\mathscr{A}$ (since $\left\|T S_{\alpha}\right\|_{\mathscr{A}} \leq\|T\|_{\mathscr{A}}\left\|S_{\alpha}\right\|$ ), and it is equivalent to the $\lambda$-BAP for the ideal $\mathscr{L}$ of all bounded linear operators.

By [17] (see [20] for a simpler proof), the weak $\lambda$-BAP and the $\lambda$-BAP are equivalent for a Banach space $X$ whenever $X^{*}$ or $X^{* *}$ has the Radon-Nikodým property. It remains open whether the weak $\lambda$-BAP is strictly weaker than the $\lambda$-BAP. If they were equivalent, then, by [12], the answer to the long-standing famous open problem (Problem 3.8 in [1]), whether the AP of a dual Banach space implies the 1-BAP, would be "yes". For a recent survey on bounded approximation properties, see [21].

In [11], it was proved that the BAP is precisely the BAP for the ideal $\mathscr{I}$ of integral operators, and the weak BAP is precisely the BAP for the ideal $\mathscr{N}$ of nuclear operators. In [11], it was also proved that in these cases the requirement "for every Banach space $Y$ " can be relaxed by taking $Y=\ell_{\infty}^{*}$ for the BAP and $Y=c_{0}^{*}$ for the weak BAP. More precisely, the following holds.

Theorem 1.1 (see [11, Theorem 2.1 and Proposition 4.2]). Let $X$ be a Banach space, and let $1 \leq \lambda<\infty$. The following statements are equivalent.

(a) $X$ has the $\lambda$-BAP.

(b) For every Banach space $Y$ and every operator $T \in \mathscr{I}(X, Y)$ there exists a net $\left(S_{\alpha}\right) \subset \mathscr{F}(X)$ such that $S_{\alpha} \rightarrow I_{X}$ uniformly on compact subsets of $X$ and

$$
\lim \sup \left\|T S_{\alpha}\right\|_{\mathscr{I}} \leq \lambda\|T\|_{\mathscr{I}} .
$$

$\alpha$

(c) For every $T \in \mathscr{I}\left(X, \ell_{\infty}^{*}\right)$ there exists a net $\left(S_{\alpha}\right) \subset \mathscr{F}(X)$ such that $S_{\alpha} \rightarrow I_{X}$ pointwise and

$$
\limsup _{\alpha}\left\|T S_{\alpha}\right\|_{\mathscr{I}} \leq \lambda\|T\|_{\mathscr{I}} .
$$

Theorem 1.2 (see [11, Theorem 3.1 and Proposition 4.1]). Let $X$ be a Banach space, and let $1 \leq \lambda<\infty$. The following statements are equivalent.

(a) $X$ has the weak $\lambda-B A P$.

(b) For every Banach space $Y$ and every operator $T \in \mathscr{N}(X, Y)$ there exists a net $\left(S_{\alpha}\right) \subset \mathscr{F}(X)$ such that $S_{\alpha} \rightarrow I_{X}$ uniformly on compact subsets of $X$ and

$$
\underset{\alpha}{\lim \sup }\left\|T S_{\alpha}\right\|_{\mathcal{N}} \leq \lambda\|T\|_{\mathcal{N}} .
$$


(c) For every $T \in \mathcal{N}\left(X, c_{0}^{*}\right)$ there exists a net $\left(S_{\alpha}\right) \subset \mathscr{F}(X)$ such that $S_{\alpha} \rightarrow I_{X}$ pointwise and

$$
\limsup _{\alpha}\left\|T S_{\alpha}\right\|_{\mathcal{N}} \leq \lambda\|T\|_{\mathcal{N}} .
$$

The classical spaces $c_{0}$ and $\ell_{\infty}$ are, indeed, very different from each other. A natural question would be: can the spaces $c_{0}$ and $\ell_{\infty}$ be replaced by one classical Banach space, preferably separable, which would characterize both the BAP and the weak BAP? Our main aim of this paper is to show that the space $C[0,1]$ of continuous functions fits for the both BAPs. Our main results are as follows (conditions (b) below are to be compared with conditions (c) of Theorems 1.1 and 1.2).

Theorem 1.3. Let $X$ be a Banach space, and let $1 \leq \lambda<\infty$. The following statements are equivalent.

(a) $X$ has the $\lambda$-BAP.

(b) For every $T \in \mathscr{I}\left(X, C[0,1]^{*}\right)$ there exists a net $\left(S_{\alpha}\right) \subset \mathscr{F}(X)$ such that $S_{\alpha} \rightarrow I_{X}$ pointwise and

$$
\limsup _{\alpha}\left\|T S_{\alpha}\right\|_{\mathscr{I}} \leq \lambda\|T\|_{\mathscr{I}}
$$

Theorem 1.4. Let $X$ be a Banach space, and let $1 \leq \lambda<\infty$. The following statements are equivalent.

(a) $X$ has the weak $\lambda$-BAP.

(b) For every $T \in \mathcal{N}\left(X, C[0,1]^{*}\right)$ there exists a net $\left(S_{\alpha}\right) \subset \mathscr{F}(X)$ such that $S_{\alpha} \rightarrow I_{X}$ pointwise and

$$
\limsup _{\alpha}\left\|T S_{\alpha}\right\|_{\mathcal{N}} \leq \lambda\|T\|_{\mathcal{N}} .
$$

Theorem 1.4 and the separable case of Theorem 1.3 will be proved in Section 2 relying on the fact that the Banach operator ideal $\mathscr{I}$ is injective with respect to norm-preserving extension operators (see Proposition 2.1). The nonseparable case of Theorem 1.3 will be deduced from the separable case in Section 3 relying on the main result of Section 3 (Theorem 3.2) stating that a property of $X$, similar to conditions (c) of Theorems 1.1 and 1.2 and to conditions (b) of Theorems 1.3 and 1.4, is inherited by ideals in Banach spaces.

Our notation is standard. A Banach space $X$ will be regarded as a subspace of its bidual $X^{* *}$ under the canonical embedding $j_{X}: X \rightarrow X^{* *}$. The closure of a set $A \subset X$ is denoted $\bar{A}$. The tensor product $X \otimes Y$ with a tensor norm 
$\alpha$ is denoted by $X \otimes_{\alpha} Y$ and its completion by $X \hat{\otimes}_{\alpha} Y$. We shall use only the classical projective tensor norm $\pi=\|\|_{\pi}$ and the injective tensor norm $\varepsilon$. Since $\mathscr{F}(X, Y)=X^{*} \otimes Y$, we shall write $\|T\|_{\pi}$ for $T \in \mathscr{F}(X, Y)\left(\|\|_{\pi}\right.$ is called the finite nuclear norm in [22]). Let us recall that, for Banach operator ideals $\mathscr{A}$ and $\mathscr{B}$, the inclusion $\mathscr{A} \subset \mathscr{B}$ means that $\mathscr{A}(X, Y) \subset \mathscr{B}(X, Y)$ and $\|T\|_{\mathscr{A}} \geq\|T\|_{\mathscr{B}}$ for all Banach spaces $X$ and $Y$ and for all operators $T \in \mathscr{A}(X, Y)$.

We refer to the books by Diestel and Uhl [3] and Ryan [23] for the classical approximation properties, tensor products, and for the common Banach operator ideals such as $\mathscr{N}$ and $\mathscr{I}$; see also [2] by Diestel, Jarchow, and Tonge and Pietsch's book [22] for operator ideals. We use "Banach operator ideal" for "normed operator ideal" in [22], or for "Banach ideal" in [2] and [23] (note that, in the Banach spaces context, the term "ideal" has its own meaning (see Section 2)).

\section{Proofs of Theorem 1.4 and the separable case of Theorem 1.3}

Recall that a Banach operator ideal $\mathscr{A}$ is injective if $\|J T\|_{\mathscr{A}}=\|T\|_{\mathscr{A}}$ whenever $T \in \mathscr{A}(X, Y)$ and $J \in \mathscr{L}(Y, Z)$ is an into isometry. It is well known that the Banach operator ideal $\mathscr{I}$ of integral operators is not injective (see, e.g., $[22,8.4 .10])$. Our first result shows that $\mathscr{I}$ is injective with respect to normpreserving extension operators, a fact which will be used in the proofs of Theorems 1.4 and 2.6 below.

Let $Y$ be a closed subspace of a Banach space $Z$. An operator $\Phi \in \mathscr{L}\left(Y^{*}, Z^{*}\right)$ is called an extension operator if $\left(\Phi y^{*}\right)(y)=y^{*}(y)$ for all $y^{*} \in Y^{*}$ and all $y \in Y$. If $Y$ admits an extension operator $\Phi \in \mathscr{L}\left(Y^{*}, Z^{*}\right)$, which is normpreserving (i.e., $\|\Phi\|=1$ ), then $Y$ is called an ideal in $Z$. This is equivalent to the annihilator $Y^{\perp}$ of $Y$ being the kernel of a norm one projection in $Z^{*}$.

Proposition 2.1. Let $X$ be a Banach space. Let $Y$ be a closed subspace of a Banach space $Z$. If there exists a norm-preserving extension operator $\Phi \in \mathscr{L}\left(Y^{*}, Z^{*}\right)$, then $\|\Phi T\|_{\mathscr{I}}=\|T\|_{\mathscr{I}}$ whenever $T \in \mathscr{I}\left(X, Y^{*}\right)$.

Proof. We are going to use well-known facts about tensor products (see, e.g., [3] or [23]). Since $\mathscr{I}\left(X, Y^{*}\right)=\left(X \otimes_{\varepsilon} Y\right)^{*}$ and $\mathscr{I}\left(X, Z^{*}\right)=\left(X \otimes_{\varepsilon} Z\right)^{*}$, we may consider $T \in\left(X \otimes_{\varepsilon} Y\right)^{*}$ and $\Phi T \in\left(X \otimes_{\varepsilon} Z\right)^{*}$. Taking into account that $X \otimes_{\varepsilon} Y$ is a subspace of $X \otimes_{\varepsilon} Z$, let us observe that $\Phi T$ extends $T$. Indeed, for all $x \in X$ and $y \in Y$,

$$
(\Phi T)(x \otimes y)=(\Phi T x)(y)=(T x)(y)=T(x \otimes y) .
$$

Hence, $\|\Phi T\|_{\mathscr{I}} \geq\|T\|_{\mathscr{I}}$. On the other hand, $\|\Phi T\|_{\mathscr{I}} \leq\|\Phi\|\|T\|_{\mathscr{I}}=\|T\|_{\mathscr{I}}$. 
Recall that a Banach space is a $\mathscr{P}_{\lambda}$-space, for some $\lambda \geq 1$, if it is complemented, by a projection whose norm does not exceed $\lambda$, in any Banach space containing it (as an isometrically isomorphic subspace). The next result is due to Fakhoury [4, Corollary 3.3]. Fakhoury's proof relies on Lindenstrauss's Memoir [14] and his own results established in [4]. For a simple direct proof, see [16, Proposition 5.3].

Proposition 2.2. Let $Y$ be a closed subspace of a Banach space Z. If $Y^{* *}$ is a $\mathscr{P}_{\lambda}$-space, then there exists an extension operator $\Phi \in \mathscr{L}\left(Y^{*}, Z^{*}\right)$ with $\|\Phi\| \leq \lambda$.

It is well known that, for every set $\Gamma$, the space $\ell_{\infty}(\Gamma)$ is a $\mathscr{P}_{1}$-space (see, e.g., $\left[15\right.$, p. 105]). In particular, $c_{0}^{* *}=\ell_{\infty}$ is a $\mathscr{P}_{1}$-space. More generally, $Y^{* *}$ is a $\mathscr{P}_{1}$-space whenever $Y$ is an $L_{1}$-predual, i.e., $Y^{*}$ is isometrically isomorphic to a space of type $L_{1}(\Omega, \mu)$ (see, e.g., [26, p. 1706]).

Corollary 2.3. Let $Y$ be an $L_{1}$-predual (in particular, $Y=c_{0}$ ). If $Y$ is contained in a Banach space $Z$ (as an isometrically isomorphic subspace), then $Y$ is an ideal in $Z$.

On the other hand, the following holds.

Proposition 2.4 (see [4, Proposition 3.4]). Every ideal in an $L_{1}$-predual is an $L_{1}$-predual itself.

Proof. Since [4] considers only the real case and does not provide a proof, we include a proof for completeness. Thus, let $Y$ be an ideal in an $L_{1}$-predual $Z$, and let $\Phi \in \mathscr{L}\left(Y^{*}, Z^{*}\right)$ be a norm-preserving extension operator. Since $Z^{* *}$ is a $\mathscr{P}_{1}$-space and $\Phi^{*}$ provides a norm one projection in $Z^{* *}$ onto $Y^{* *}$, $Y^{* *}$ is also a $\mathscr{P}_{1}$-space (it is easily seen that 1-complemented subspaces of a $\mathscr{P}_{1}$-space are $\mathscr{P}_{1}$-spaces). Hence, by the Grothendieck-Sakai theorem (see [5] for the real case and [24] for the complex case), $Y$ is an $L_{1}$-predual.

Let us first prove Theorem 1.4.

Proof of Theorem 1.4. By Theorem 1.2, we only need to prove the implication (b) $\Rightarrow$ (a). For this, it suffices to show that condition (b) of Theorem 1.4 implies condition (c) of Theorem 1.2.

Let $T \in \mathscr{N}\left(X, c_{0}^{*}\right)$. Since $c_{0}$ embeds isometrically in $C[0,1]$, by Corollary 2.3 there exists a norm-preserving extension operator $\Phi \in \mathscr{L}\left(c_{0}^{*}, C[0,1]^{*}\right)$. Since $\Phi T \in \mathscr{N}\left(X, C[0,1]^{*}\right)$, there exists $\left(S_{\alpha}\right) \subset \mathscr{F}(X)$ such that $S_{\alpha} \rightarrow I_{X}$ pointwise and

$$
\underset{\alpha}{\lim \sup }\left\|\Phi T S_{\alpha}\right\|_{\mathcal{H}} \leq \lambda\|\Phi T\|_{\mathcal{N}} \leq \lambda\|\Phi\|\|T\|_{\mathcal{N}}=\lambda\|T\|_{\mathcal{N}} .
$$


It is well known (see, e.g., [23, p. 176]) that for a finite-rank operator, acting to a space with the metric AP, its nuclear and integral norms coincide. Hence, $\left\|T S_{\alpha}\right\|_{\mathcal{H}}=\left\|T S_{\alpha}\right\|_{\mathscr{S}}$ and $\left\|\Phi T S_{\alpha}\right\|_{\mathcal{H}}=\left\|\Phi T S_{\alpha}\right\|_{\mathscr{S}}$. Using Proposition 2.1, we therefore have

$$
\left\|T S_{\alpha}\right\|_{\mathcal{H}}=\left\|T S_{\alpha}\right\|_{\mathscr{I}}=\left\|\Phi T S_{\alpha}\right\|_{\mathscr{I}}=\left\|\Phi T S_{\alpha}\right\|_{\mathcal{N}} .
$$

Hence,

$$
\limsup _{\alpha}\left\|T S_{\alpha}\right\|_{\mathcal{N}} \leq \lambda\|T\|_{\mathcal{N}}
$$

as desired.

REMARK 2.5. It is an easy exercise to show that $c_{0}^{*}=\ell_{1}$ embeds isometrically in $C[0,1]^{*}$. It seems that an arbitrary into isometry $\Phi \in \mathscr{L}\left(c_{0}^{*}, C[0,1]^{*}\right)$ cannot be used for proving Theorem 1.4.

The separable case of Theorem 1.3 is immediate from Theorem 2.6 below and Theorem 1.1.

Theorem 2.6. Let $X$ be a separable Banach space, and let $1 \leq \lambda<\infty$. If for every $T \in \mathscr{I}\left(X, C[0,1]^{*}\right)$ there exists a net $\left(S_{\alpha}\right) \subset \mathscr{F}(X)$ such that $S_{\alpha} \rightarrow I_{X}$ uniformly on compact subsets of $X$ (respectively, pointwise) and

$$
\limsup _{\alpha}\left\|T S_{\alpha}\right\|_{\mathscr{I}} \leq \lambda\|T\|_{\mathscr{I}}
$$

then for every $T \in \mathscr{I}\left(X, \ell_{\infty}^{*}\right)$ there exists a net $\left(S_{\alpha}\right) \subset \mathscr{F}(X)$ with the same properties.

Proof. Let $T \in \mathscr{I}\left(X, \ell_{\infty}^{*}\right)$. Since ran $T$ is separable, by a result of Sims and Yost [25] (see [6, p. 138]), we can find a separable ideal $Y$ in $\ell_{\infty}$ which admits a norm-preserving extension operator $\Phi \in \mathscr{L}\left(Y^{*}, \ell_{\infty}^{*}\right)$ satisfying $\operatorname{ran} T \subset$ ran $\Phi$. By Proposition 2.4, $Y$ is an $L_{1}$-predual.

Let $j: Y \rightarrow \ell_{\infty}$ denote the identity embedding. Observe that

$$
T=\Phi j^{*} T .
$$

Indeed, let $x \in X$. Since $\operatorname{ran} T \subset \operatorname{ran} \Phi$, there is $y^{*} \in Y^{*}$ such that $T x=\Phi y^{*}$. Hence, $\Phi j^{*} T x=\Phi j^{*} \Phi y^{*}=\Phi I_{Y^{*}} y^{*}=\Phi y^{*}=T x$.

Since $Y$ is separable, it embeds isometrically in $C[0,1]$. By Corollary 2.3, there exists a norm-preserving extension operator $\Psi \in \mathscr{L}\left(Y^{*}, C[0,1]^{*}\right)$. Since $\Psi j^{*} T \in \mathscr{I}\left(X, C[0,1]^{*}\right)$, there exists a net $\left(S_{\alpha}\right) \subset \mathscr{F}(X)$ such that $S_{\alpha} \rightarrow I_{X}$ and

$$
\limsup _{\alpha}\left\|\Psi j^{*} T S_{\alpha}\right\|_{\mathscr{I}} \leq \lambda\left\|\Psi j^{*} T\right\|_{\mathscr{I}} \leq \lambda\|T\|_{\mathscr{I}} .
$$


On the other hand, using Proposition 2.1 twice, we have

$$
\left\|T S_{\alpha}\right\|_{\mathscr{I}}=\left\|\Phi j^{*} T S_{\alpha}\right\|_{\mathscr{I}}=\left\|j^{*} T S_{\alpha}\right\|_{\mathscr{I}}=\left\|\Psi j^{*} T S_{\alpha}\right\|_{\mathscr{I}} .
$$

From this, the desired inequality is immediate.

Remark 2.7. In the above proof of Theorem 2.6, we applied Proposition 2.4 to show that an ideal $Y$ in $\ell_{\infty}$ is an $L_{1}$-predual. An alternative proof of this fact, relying on intersection properties of balls, can be done as follows. By results of Lindenstrauss [14] (the real case) and Hustad [8] (the complex case) (see [9, Theorem 4.1] and [10, Theorem 5.8]), $Y$ is an $L_{1}$-predual if and only if $Y$ is an almost $E(n)$-space for all $n \in \mathrm{N}$. Recall (see [8] and [10, p. 9]) that a Banach space $Y$ is an almost $E(n)$ space if for each family of $n$ closed balls $B\left(y_{1}, r_{1}\right), \ldots, B\left(y_{n}, r_{n}\right)$ in $Y$ the following implication holds:

$$
\begin{aligned}
& \bigcap_{i=1}^{n} B\left(y^{*}\left(y_{i}\right), r_{i}\right) \neq \varnothing \quad \forall y^{*} \in Y^{*},\left\|y^{*}\right\| \leq 1 \\
\Rightarrow \bigcap_{i=1}^{n} B\left(y_{i}, r_{i}+\varepsilon\right) \neq \varnothing & \forall \varepsilon>0 .
\end{aligned}
$$

Let $e_{k}^{*} \in \ell_{\infty}^{*}$ be the coordinate functionals, and let $y_{k}^{*} \in Y^{*}$ be their restrictions to $Y$. If the above assumption holds, then there exist numbers $a_{k}$ such that $\left|e_{k}^{*}\left(y_{i}\right)-a_{k}\right|=\left|y_{k}^{*}\left(y_{i}\right)-a_{k}\right| \leq r_{i}$ for all $i=1, \ldots, n$. Hence, $x:=\left(a_{k}\right) \in \ell_{\infty}$ and $\left\|y_{i}-x\right\| \leq r_{i}$ in $\ell_{\infty}$ for all $i=1, \ldots, n$. But then $\left\|y_{i}-\Phi^{*} x\right\|=\| \Phi^{*}\left(y_{i}-\right.$ $x) \| \leq r_{i}$ in $Y^{* *}$ for all $i=1, \ldots, n$. This implies, by the principle of local reflexivity, that for every $\varepsilon>0$ there exists $y_{\varepsilon} \in Y$ such that $\left\|y_{i}-y_{\varepsilon}\right\| \leq r_{i}+\varepsilon$ for all $i=1, \ldots, n$, as desired.

\section{Proof of the non-separable case of Theorem 1.3}

The proof of the non-separable case of Theorem 1.3 relies on the following reformulation of the BAP in terms of separable ideals.

Theorem 3.1 (see [11, Proposition 4.3 and Theorem 2.2]). Let $X$ be a Banach space, and let $1 \leq \lambda<\infty$. The following statements are equivalent.

(a) $X$ has the $\lambda$-BAP.

(b) Every separable ideal $Z$ in $X$ has the $\lambda-B A P$.

The next result is the main theorem of this section. Its assumption $\mathscr{A} \subset \mathscr{W}$ can be equivalently expressed as follows: if $T \in \mathscr{A}(X, Y)$, then $\operatorname{ran} T^{* *} \subset Y$. This assumption holds for many operator ideals. For us, it is important that $\mathscr{I} \subset \mathscr{W}$. 
THeORem 3.2. Let $X$ and $Y$ be Banach spaces, let $\mathscr{A}$ be a Banach operator ideal such that $\mathscr{A} \subset \mathscr{W}$, and let $1 \leq \lambda<\infty$. Assume that $X$ has the weak $B A P$. If $X$ has the property that for every $T \in \mathscr{A}\left(Y, X^{*}\right)$ there exists a net $\left(S_{\alpha}\right) \subset \mathscr{F}(X)$ such that $S_{\alpha} \rightarrow I_{X}$ pointwise and

$$
\underset{\alpha}{\lim \sup }\left\|S_{\alpha}^{*} T\right\|_{\mathscr{A}} \leq \lambda\|T\|_{\mathscr{A}}
$$

then every ideal $Z$ in $X$ has the same property.

Proof. Let $T \in \mathscr{A}\left(Y, Z^{*}\right)$. We consider the set of all $v=(\varepsilon, K, L)$, where $\varepsilon>0$, and $K \subset Z$ and $L \subset Z^{*}$ are finite sets. We need to prove that for every $v=(\varepsilon, K, L)$ there exists $U_{v} \in \mathscr{F}(Z)$ such that

$$
\left|z^{*}\left(U_{v} z-z\right)\right|<\varepsilon \quad \forall z \in K, \quad \forall z^{*} \in L
$$

and

$$
\left\|U_{v}^{*} T\right\|_{\mathscr{A}} \leq \lambda\|T\|_{\mathscr{A}}+\varepsilon
$$

Indeed, this would imply that $U_{v} \rightarrow I_{Z}$ in the weak operator topology and

$$
\limsup _{v}\left\|U_{v}^{*} T\right\|_{\mathscr{A}} \leq \lambda\|T\|_{\mathscr{A}}
$$

Hence, passing to a net of convex combinations far out in $\left(U_{v}\right)$, we could assume that $U_{v} \rightarrow I_{Z}$ in the strong operator topology, as desired.

Let us fix $v=(\varepsilon, K, L)$. Let $\Phi \in \mathscr{L}\left(Z^{*}, X^{*}\right)$ be a norm-preserving extension operator. Then $\Phi T \in \mathscr{A}\left(Y, X^{*}\right)$, and there exists $S=S_{\alpha} \in \mathscr{F}(X)$ such that

$$
\|S z-z\|<\frac{\varepsilon}{2 \max \left\{\left\|z^{*}\right\|: z^{*} \in L\right\}} \quad \forall z \in K,
$$

and

$$
\left\|S^{*} \Phi T\right\|_{\mathscr{A}} \leq \lambda\|\Phi T\|_{\mathscr{A}}+\frac{\varepsilon}{2} \leq \lambda\|T\|_{\mathscr{A}}+\frac{\varepsilon}{2}
$$

Since $X$ has the weak BAP, there exists an extension operator $\Psi \in \overline{X \otimes X^{*}} w^{*}$ $\subset \mathscr{L}\left(X^{*}, X^{* * *}\right)=\left(X^{*} \hat{\otimes}_{\pi} X^{* *}\right)^{*}$ (see [13, Propositions 2.1, 2.3, and 2.5] and [20, Corollary 3.18]). Then $\Psi \Phi \in \mathscr{L}\left(Z^{*}, X^{* * *}\right)=\left(Z^{*} \hat{\otimes}_{\pi} X^{* *}\right)^{*}$. We show that $\Psi \Phi \in \overline{Z \otimes X^{*}} w^{*}$. Let $u=\sum_{n=1}^{\infty} z_{n}^{*} \otimes x_{n}^{* *} \in Z^{*} \hat{\otimes}_{\pi} X^{* *}$, with $\sum_{n=1}^{\infty}\left\|z_{n}^{*}\right\|\left\|x_{n}^{* *}\right\|<\infty$, and assume that

$$
\left\langle u, z \otimes x^{*}\right\rangle=\sum_{n=1}^{\infty} z_{n}^{*}(z) x_{n}^{* *}\left(x^{*}\right)=0 \quad \forall z \in Z, \quad \forall x^{*} \in X^{*} .
$$


This means that $\sum_{n=1}^{\infty} x_{n}^{* *}\left(x^{*}\right) z_{n}^{*}=0$ in $Z^{*}$ for all $x^{*} \in X^{*}$, and therefore

$$
\sum_{n=1}^{\infty} x_{n}^{* *}\left(x^{*}\right) \Phi z_{n}^{*}=0 \quad \forall x^{*} \in X^{*}
$$

in $X^{*}$. Hence, denoting $v=\sum_{n=1}^{\infty} \Phi z_{n}^{*} \otimes x_{n}^{* *} \in X^{*} \hat{\otimes}_{\pi} X^{* *}$, we have

$$
\langle u, \Psi \Phi\rangle=\sum_{n=1}^{\infty}\left(\Psi \Phi z_{n}^{*}\right)\left(x_{n}^{* *}\right)=\langle v, \Psi\rangle=0,
$$

because

$$
\begin{aligned}
\left\langle v, x \otimes x^{*}\right\rangle & =\sum_{n=1}^{\infty}\left(\Phi z_{n}^{*}\right)(x) x_{n}^{* *}\left(x^{*}\right) \\
& =\left(\sum_{n=1}^{\infty} x_{n}^{* *}\left(x^{*}\right) \Phi z_{n}^{*}\right)(x)=0 \quad \forall x \in X, \quad \forall x^{*} \in X^{*} .
\end{aligned}
$$

Since $\Psi \Phi \in \overline{Z \otimes X^{*}} w^{*}$, there exists a net $\left(V_{\beta}\right) \subset \mathscr{F}(X, Z)$ such that $V_{\beta}^{*} \rightarrow \Psi \Phi$ weak* in $\mathscr{L}\left(Z^{*}, X^{* * *}\right)=\left(Z^{*} \hat{\otimes}_{\pi} X^{* *}\right)^{*}$. We shall show that the desired operator $U_{v}$ can be found in the form $U_{v}=V S i_{Z}$, where $i_{Z}: Z \rightarrow X$ denotes the identity embedding and $V$ is a convex combination of operators $V_{\beta}$.

Set $H=\operatorname{ran}\left(i_{Z}^{*} S^{*}\right)$. Then $\operatorname{dim} H<\infty$. Let $i_{H}: H \rightarrow Z^{*}$ be the identity embedding. Denote by $\hat{S}$ the operator $i_{Z}^{*} S^{*}$ considered as an operator to $H$. Then

$$
i_{H} \hat{S}=i_{Z}^{*} S^{*}
$$

and the operators $\hat{S} V_{\beta}^{*} T$ and $\hat{S}^{* *} \Psi \Phi T$ belong to $\mathscr{F}(Y, H)=Y^{*} \otimes H$. Since $\left(Y^{*} \hat{\otimes}_{\pi} H\right)^{*}=\mathscr{L}\left(Y^{*}, H^{*}\right)=\mathscr{F}\left(Y^{*}, H^{*}\right)=Y^{* *} \otimes H^{*}$ and we have (using that $\left.\operatorname{ran} T^{* *} \subset Z^{*}\right)$ that for all $y^{* *} \in Y^{* *}$ and $h^{*} \in H^{*}$

$$
\begin{aligned}
& \left\langle y^{* *} \otimes h^{*}, \hat{S} V_{\beta}^{*} T\right\rangle=h^{*}\left(\hat{S}^{* *} V_{\beta}^{* * *} T^{* *} y^{* *}\right)=h^{*}\left(\hat{S} V_{\beta}^{*} T^{* *} y^{* *}\right) \\
& \quad=\left(\hat{S}^{*} h^{*}\right)\left(V_{\beta}^{*} T^{* *} y^{* *}\right)_{\beta}^{\rightarrow}\left\langle T^{* *} y^{* *} \otimes \hat{S}^{*} h^{*}, \Psi \Phi\right\rangle=\left\langle y^{* *} \otimes h^{*}, \hat{S}^{* *} \Psi \Phi T\right\rangle,
\end{aligned}
$$

the net $\left(\hat{S} V_{\beta}^{*} T\right)_{\beta}$ converges to $\hat{S}^{* *} \Psi \Phi T$ weakly in $Y^{*} \hat{\otimes}_{\pi} H$. Passing to a net of convex combinations far out in $\left(V_{\beta}\right)$, we may assume that our net $\left(V_{\beta}\right)$ also satisfies

$$
\left\|\hat{S} V_{\beta}^{*} T-\hat{S}^{* *} \Psi \Phi T\right\|_{\pi} \underset{\beta}{\rightarrow} 0,
$$


hence also

$$
\left\|i_{H} \hat{S} V_{\beta}^{*} T-i_{H} \hat{S}^{* *} \Psi \Phi T\right\|_{\mathscr{A}} \underset{\beta}{\rightarrow} 0
$$

Consequently,

$$
\left\|\left(V_{\beta} S i_{Z}\right)^{*} T\right\|_{\mathscr{A}}=\left\|i_{H} \hat{S} V_{\beta}^{*} T\right\|_{\mathscr{A}} \underset{\beta}{\rightarrow}\left\|i_{H} \hat{S}^{* *} \Psi \Phi T\right\|_{\mathscr{A}} .
$$

A straightforward calculation shows that $i_{H} \hat{S}^{* *}=i_{Z}^{*} S^{*} j_{X}^{*}$. Since $j_{X}^{*} \Psi=I_{X^{*}}$,

$$
\begin{aligned}
\left\|i_{H} \hat{S}^{* *} \Psi \Phi T\right\|_{\mathscr{A}} & =\left\|i_{Z}^{*} S^{*} j_{X}^{*} \Psi \Phi T\right\|_{\mathscr{A}}=\left\|i_{Z}^{*} S^{*} \Phi T\right\|_{\mathscr{A}} \\
& \leq\left\|S^{*} \Phi T\right\|_{\mathscr{A}} \leq \lambda\|T\|_{\mathscr{A}}+\frac{\varepsilon}{2} .
\end{aligned}
$$

Hence, there is some $\beta_{0}$ such that for $\beta \geq \beta_{0}$, one has

$$
\left\|\left(V_{\beta} S i_{Z}\right)^{*} T\right\|_{\mathscr{A}} \leq \lambda\|T\|_{\mathscr{A}}+\varepsilon .
$$

Finally, let us consider the operators $\hat{S} V_{\beta}^{*}, \hat{S}^{* *} \Psi \Phi \in \mathscr{F}\left(Z^{*}, H\right)$. Since for all $z^{*} \in Z^{*}$ and $h^{*} \in H^{*}$

$$
h^{*}\left(\hat{S} V_{\beta}^{*} z^{*}\right)=\left(\hat{S}^{*} h^{*}\right)\left(V_{\beta}^{*} z^{*}\right) \underset{\beta}{\rightarrow}\left\langle z^{*} \otimes \hat{S}^{*} h^{*}, \Psi \Phi\right\rangle=h^{*}\left(\hat{S}^{* *} \Psi \Phi z^{*}\right),
$$

$\hat{S} V_{\beta}^{*} \rightarrow{ }_{\beta} \hat{S}^{* *} \Psi \Phi$ in the weak operator topology. Passing to a net of convex combinations far out in $\left(\hat{S} V_{\beta}^{*}\right)$, we may assume that $\hat{S} V_{\beta}^{*} z^{*} \rightarrow_{\beta} \hat{S}^{* *} \Psi \Phi z^{*}$ for all $z^{*} \in Z^{*}$. Hence also $i_{H} \hat{S} V_{\beta}^{*} z^{*} \rightarrow_{\beta} i_{H} \hat{S}^{* *} \Psi \Phi z^{*}$ for all $z^{*} \in Z^{*}$. This means, by calculations made above, that

$$
\left(V_{\beta} S i_{Z}\right)^{*} z^{*} \underset{\beta}{\rightarrow} i_{Z}^{*} S^{*} \Phi z^{*} \quad \forall z^{*} \in Z^{*} .
$$

Let $\beta_{1}$ be such that for $\beta \geq \beta_{1}$, one has

$$
\left|z^{*}\left(V_{\beta} S i_{Z} z\right)-\left(\Phi^{*} S z\right)\left(z^{*}\right)\right|<\frac{\varepsilon}{2} \quad \forall z \in K, \quad \forall z^{*} \in L .
$$

Since

$$
\left\|\Phi^{*} S z-\Phi^{*} z\right\| \leq\|S z-z\|<\frac{\varepsilon}{2 \max \left\{\left\|z^{*}\right\|: z^{*} \in L\right\}} \quad \forall z \in K,
$$

and $\left(\Phi^{*} z\right)\left(z^{*}\right)=\left(\Phi z^{*}\right)(z)=z^{*}(z)$,

$$
\left|\left(\Phi^{*} S z\right)\left(z^{*}\right)-z^{*}(z)\right|<\frac{\varepsilon}{2} \quad \forall z \in K, \quad \forall z^{*} \in L .
$$


Consequently, for $\beta \geq \beta_{1}$, one has

$$
\left|z^{*}\left(V_{\beta} S i_{Z} z-z\right)\right|<\varepsilon \quad \forall z \in K, \quad \forall z^{*} \in L .
$$

Setting $U_{v}=V_{\beta} S i_{Z}$ for some $\beta \geq \beta_{0}, \beta \geq \beta_{1}$, completes the proof.

To apply Theorem 3.2 in our context, we shall need the following result. For a Banach operator ideal $\mathscr{A}$, let us denote by $\mathscr{A}^{*}$ the dual operator ideal of $\mathscr{A}$. Its components are $\mathscr{A}^{*}(X, Y)=\left\{T \in \mathscr{L}(X, Y): T^{*} \in \mathscr{A}\left(Y^{*}, X^{*}\right)\right\}$ with $\|T\|_{\mathscr{A}^{*}}=\left\|T^{*}\right\|_{\mathscr{A}}$. (The notation $\mathscr{A}^{*}$ means adjoint ideal in [2] and [22], where the dual operator ideal is denoted by $\mathscr{A}^{d}$ and $\mathscr{A}^{\text {dual }}$, respectively.)

Proposition 3.3. Let $X$ and $Y$ be Banach spaces, let $\mathscr{A}$ be a Banach operator ideal such that $\mathscr{A} \subset \mathscr{A}^{* *}$, and let $1 \leq \lambda<\infty$. Let $\tau$ be a topology on $\mathscr{L}(X)$. The following statements are equivalent.

(a) For every $T \in \mathscr{A}\left(Y, X^{*}\right)$ there exists a net $\left(S_{\alpha}\right) \subset \mathscr{F}(X)$ such that $S_{\alpha} \rightarrow I_{X}$ in $\tau$ and

$$
\limsup _{\alpha}\left\|S_{\alpha}^{*} T\right\|_{\mathscr{A}} \leq \lambda\|T\|_{\mathscr{A}} .
$$

(b) For every $T \in \mathscr{A}^{*}\left(X, Y^{*}\right)$ there exists a net $\left(S_{\alpha}\right) \subset \mathscr{F}(X)$ such that $S_{\alpha} \rightarrow I_{X}$ in $\tau$ and

$$
\lim \sup \left\|T S_{\alpha}\right\|_{\mathscr{A}^{*}} \leq \lambda\|T\|_{\mathscr{A}^{*}} .
$$

Proof. Below, we shall use the following observation. If $X$ and $Y$ are Banach spaces and $T \in \mathscr{L}\left(X, Y^{*}\right)$, then

$$
T=j_{Y}^{*} T^{* *} j_{X} .
$$

Indeed, $T=I_{Y^{*}} T=j_{Y}^{*} j_{Y^{*}} T=j_{Y}^{*} T^{* *} j_{X}$.

(a) $\Rightarrow$ (b). Consider $T \in \mathscr{A}^{*}\left(X, Y^{*}\right)$. Then $T^{*} \in \mathscr{A}\left(Y^{* *}, X^{*}\right)$ and $\|T\|_{\mathscr{A}^{*}}=$ $\left\|T^{*}\right\|_{\mathscr{A}}$. Since $T^{*} j_{Y} \in \mathscr{A}\left(Y, X^{*}\right)$, there is $\left(S_{\alpha}\right) \subset \mathscr{F}(X)$ such that $S_{\alpha} \rightarrow I_{X}$ in $\tau$ and

$$
\limsup _{\alpha}\left\|S_{\alpha}^{*} T^{*} j_{Y}\right\|_{\mathscr{A}} \leq \lambda\left\|T^{*} j_{Y}\right\|_{\mathscr{A}} \leq \lambda\left\|T^{*}\right\|_{\mathscr{A}}=\lambda\|T\|_{\mathscr{A}^{*}} .
$$

On the other hand,

$$
\begin{aligned}
\left\|T S_{\alpha}\right\|_{\mathscr{A}^{*}} & =\left\|j_{Y}^{*} T^{* *} S_{\alpha}^{* *} j_{X}\right\|_{\mathscr{A}^{*}} \leq\left\|j_{Y}^{*} T^{* *} S_{\alpha}^{* *}\right\|_{\mathscr{A}^{*}} \\
& =\left\|S_{\alpha}^{*} T^{*} j_{Y}\right\|_{\mathscr{A}}{ }^{* *} \leq\left\|S_{\alpha}^{*} T^{*} j_{Y}\right\|_{\mathscr{A}} .
\end{aligned}
$$

From this, the desired inequality is immediate. 
(b) $\Rightarrow$ (a). Consider $T \in \mathscr{A}\left(Y, X^{*}\right)$. Since $\mathscr{A} \subset \mathscr{A}^{* *}$, we have $T \in$ $\mathscr{A}^{* *}\left(Y, X^{*}\right)$ and therefore $T^{*} \in \mathscr{A}^{*}\left(X^{* *}, Y^{*}\right)$. Since $T^{*} j_{X} \in \mathscr{A}^{*}\left(X, Y^{*}\right)$, there is $\left(S_{\alpha}\right) \subset \mathscr{F}(X)$ such that $S_{\alpha} \rightarrow I_{X}$ in $\tau$ and

$\limsup _{\alpha}\left\|T^{*} j_{X} S_{\alpha}\right\|_{\mathscr{A}^{*}} \leq \lambda\left\|T^{*} j_{X}\right\|_{\mathscr{A}^{*}} \leq \lambda\left\|T^{*}\right\|_{\mathscr{A}^{*}}=\lambda\|T\|_{\mathscr{A}^{* *}} \leq \lambda\|T\|_{\mathscr{A}}$.

On the other hand,

$$
\left\|S_{\alpha}^{*} T\right\|_{\mathscr{A}}=\left\|S_{\alpha}^{*} j_{X}^{*} T^{* *} j_{Y}\right\|_{\mathscr{A}} \leq\left\|S_{\alpha}^{*} j_{X}^{*} T^{* *}\right\|_{\mathscr{A}}=\left\|T^{*} j_{X} S_{\alpha}\right\|_{\mathscr{A}} .
$$

From this, the desired inequality is immediate.

Since $\mathscr{I}=\mathscr{I}^{*}=\mathscr{I}^{* *}$, we have an immediate corollary, which we spell out for an easy reference.

Corollary 3.4. Let $X$ and $Y$ be Banach spaces, and let $1 \leq \lambda<\infty$. Let $\tau$ be a topology on $\mathscr{L}(X)$. The following statements are equivalent.

(a) For every $T \in \mathscr{I}\left(Y, X^{*}\right)$ there exists a net $\left(S_{\alpha}\right) \subset \mathscr{F}(X)$ such that $S_{\alpha} \rightarrow I_{X}$ in $\tau$ and

$$
\lim \sup _{\alpha}\left\|S_{\alpha}^{*} T\right\|_{\mathscr{I}} \leq \lambda\|T\|_{\mathscr{I}}
$$

(b) For every $T \in \mathscr{I}\left(X, Y^{*}\right)$ there exists a net $\left(S_{\alpha}\right) \subset \mathscr{F}(X)$ such that $S_{\alpha} \rightarrow I_{X}$ in $\tau$ and

$$
\limsup _{\alpha}\left\|T S_{\alpha}\right\|_{\mathscr{I}} \leq \lambda\|T\|_{\mathscr{I}}
$$

Proof of Theorem 1.3. By Theorem 1.1, we only need to prove the implication (b) $\Rightarrow$ (a).

First of all, let us observe that $X$ has the weak $\lambda$-BAP. Indeed, (b) of Theorem 1.3 implies (b) of Theorem 1.4, because $\mathcal{N} \subset \mathscr{I}$ and, as in the proof of Theorem 1.4, $\left\|T S_{\alpha}\right\|_{\mathcal{N}}=\left\|T S_{\alpha}\right\|_{\mathscr{I}}$ whenever $T S_{\alpha} \in \mathscr{F}\left(X, C[0,1]^{*}\right)$. According to Theorem 1.4, $X$ has the weak $\lambda$-BAP.

By Corollary 3.4, (b) $\Rightarrow$ (a), and Theorem 3.2, every separable ideal $Z$ has the property that for every $T \in \mathscr{I}\left(C[0,1], Z^{*}\right)$ there exists a net $\left(S_{\alpha}\right) \subset$ $\mathscr{F}(Z)$ such that $S_{\alpha} \rightarrow I_{Z}$ pointwise and $\lim \sup _{\alpha}\left\|S_{\alpha}^{*} T\right\|_{\mathscr{I}} \leq \lambda\|T\|_{\mathscr{I}}$. By Corollary 3.4, (a) $\Rightarrow$ (b), and the separable version of Theorem 1.3, we get that every separable ideal $Z$ in $X$ has the $\lambda$-BAP. This means, according to Theorem 3.1, that $X$ has the $\lambda$-BAP. 


\section{REFERENCES}

1. Casazza, P. G., Approximation properties, pp. 271-316 in: W. B. Johnson and J. Lindenstrauss (eds.), Handbook of the Geometry of Banach Spaces 1, North-Holland, Amsterdam 2001.

2. Diestel, J., Jarchow, H., and Tonge, A., Absolutely Summing Operators, Cambridge Studies in Advanced Math. 43, Cambridge Univ. Press, Cambridge 1995.

3. Diestel, J., and Uhl, J. J. Jr, Vector Measures, Math. Surveys 15, Amer. Math. Soc., Providence, RI 1977.

4. Fakhoury, H., Sélections linéaires associées au théorème de Hahn-Banach, J. Functional Analysis 11 (1972), 436-452.

5. Grothendieck, A., Une caractérisation vectorielle métrique des espaces $L^{1}$, Canad. J. Math. 7 (1955), 552-561.

6. Harmand, P., Werner, D., and Werner, W., M-ideals in Banach Spaces and Banach Algebras, Lecture Notes in Math. 1547, Springer, Berlin 1993.

7. Heinrich, S. and Mankiewicz, P., Applications of ultrapowers to the uniform and Lipschitz classification of Banach spaces, Studia Math. 73 (1982), 225-251.

8. Hustad, O., Intersection properties of balls in complex Banach spaces whose duals are $L_{1}$ spaces, Acta Math. 132 (1974), 283-312.

9. Lima, Å., Complex Banach spaces whose duals are $L_{1}$-spaces, Israel J. Math. 24 (1976), $59-72$.

10. Lima, Å., Intersection properties of balls and subspaces in Banach spaces, Trans. Amer. Math. Soc. 227 (1977), 1-62.

11. Lima, Å., Lima, V., and Oja, E., Bounded approximation properties via integral and nuclear operators, Proc. Amer. Math. Soc. 138 (2010), 287-297.

12. Lima, Å., and Oja, E., The weak metric approximation property, Math. Ann. 333 (2005), $471-484$.

13. Lima, V., The weak metric approximation property and ideals of operators, J. Math. Anal. Appl. 334 (2007), 593-603.

14. Lindenstrauss, J., Extension of compact operators, Mem. Amer. Math. Soc. 48 (1964), 112 pp.

15. Lindenstrauss, J., and Tzafriri, L., Classical Banach Spaces I, Ergebnisse Math. Grenzgebiete 92, Springer, Berlin 1977.

16. Oja, E., Operators that are nuclear whenever they are nuclear for a larger range space, Proc. Edinburgh Math. Soc. (2) 47 (2004), 679-694.

17. Oja, E., The impact of the Radon-Nikodým property on the weak bounded approximation property, RACSAM Rev. R. Acad. Cienc. Exactas (A) 100 (2006), 325-331.

18. Oja, E., Lifting of the approximation property from Banach spaces to their dual spaces, Proc. Amer. Math. Soc. 135 (2007), 3581-3587.

19. Oja, E., The strong approximation property, J. Math. Anal. Appl. 338 (2008), 407-415.

20. Oja, E., Inner and outer inequalities with applications to approximation properties, Trans. Amer. Math. Soc. 363 (2011), 5827-5846.

21. Oja, E., On bounded approximation properties of Banach spaces, Banach Center Publ. 91 (2010), 219-231.

22. Pietsch, A., Operator Ideals, North-Holland Math. Library 20, North-Holland, Amsterdam 1980.

23. Ryan, R. A., Introduction to Tensor Products of Banach Spaces, Springer Monographs in Math., Springer, London 2002.

24. Sakai, S., $C^{*}$-algebras and $W^{*}$-algebras, Ergebnisse Math. Grenzgebiete 60, Springer, Berlin 1971.

25. Sims, B., and Yost, D., Linear Hahn-Banach extension operators, Proc. Edinburgh Math. Soc. 32 (1989), 53-57. 
26. Zippin, M., Extension of bounded linear operators, pp. 1703-1741 in: W. B. Johnson and J. Lindenstrauss (eds.), Handbook of the Geometry of Banach Spaces 2, North-Holland, Amsterdam 2003.

DEPARTMENT OF MATHEMATICS

UNIVERSITY OF AGDER

SERVICEBOKS 422

N-4604 KRISTIANSAND

NORWAY

E-mail: Asvald.Lima@uia.no

FACULTY OF MATHEMATICS AND COMPUTER SCIENCE

UNIVERSITY OF TARTU

J. LIIVI 2

EE-50409 TARTU

ESTONIA

E-mail: eve.oja@ut.ee
AALESUND UNIVERSITY COLLEGE

SERVICE BOX 17

N-6025 ÅLESUND

NORWAY

E-mail: Vegard.Lima@gmail.com 